\title{
Weighted-density approximation for general nonuniform fluid mixtures
}

\author{
Ruslan L. Davidchack and Brian B. Laird* \\ Department of Chemistry and Kansas Institute for Theoretical and Computational Science, University of Kansas,
} Lawrence, Kansas 66045

(Received 17 September 1998)

\begin{abstract}
In order to construct a general density-functional theory for nonuniform fluid mixtures, we propose an extension to multicomponent systems of the weighted-density approximation of Curtin and Ashcroft [Phys. Rev. A 32, 2909 (1985)]. This extension corrects a deficiency in a similar extension proposed earlier by Denton and Ashcroft [Phys. Rev. A 42, 7312 (1990)], in that that functional cannot be applied to the multicomponent nonuniform fluid systems with spatially varying composition, such as solid-fluid interfaces. As a test of the accuracy of our functional, we apply it to the calculation of the freezing phase diagram of a binary hard-sphere fluid, and compare the results to simulation and the Denton-Ashcroft extension. [S1063-651X(99)03509-6]

PACS number(s): 61.20.Gy, 64.70.Dv, 61.90.+d
\end{abstract}

Density-functional theory has been applied with success to the calculation of the equilibrium properties of a variety of inhomogeneous fluid systems [1]. One of the most successful of these theories has been the weighted-density approximation (WDA) of Curtin and Ashcroft [2]. The WDA has been applied with good results to problems of solid-fluid phase equilibria [2,3], solid-liquid interfaces [4,5], and nonuniform fluids [6]. For systems with well-defined bulk densities that are uniform throughout (such as in the case of freezing calculations), the modified WDA (MWDA) of Denton and Ashcroft [7-9] may be used, yielding essentially identical results as the WDA with much less computation. By construction, however, the MWDA cannot be used to study systems in which the bulk densities vary over length scales larger than a few atomic diameters (for example, interfaces or nucleation), so the full WDA must be utilized in such cases. (It is, however, often possible to exploit the system symmetry to allow for some simplification of the WDA scheme, e.g., the planar WDA of Marr and Gast [10].)

Denton and Ashcroft have proposed an extension of the WDA and MWDA for multicomponent systems [11]. Using the extended MWDA they calculated portions of the phase diagram for the binary hard-sphere system and obtained excellent agreement with the simulation results of Kranendonk and Frenkel [12]. However, the WDA that they proposed assumes that the mole fractions of the various components of the mixture are global properties of the system, making their approach inapplicable to systems, such as interfaces, in which spatially extended variations of composition may exist. In this paper, we propose a different extension of the WDA for multicomponent systems that correctly preserves the local nature of the WDA and thus can be applied to any inhomogeneous fluid system. In order to verify the validity of our approximation, we also formulate the extension of the MWDA consistent with our extension of the WDA and compare our results for freezing of a binary hard-sphere mixture with those of Denton and Ashcroft [11].

We formulate our extension of the WDA for the case of a binary mixture - the extension to three or more components

\footnotetext{
*Author to whom correspondence should be addressed.
}

is straightforward. The state of a binary mixture is specified by the single-particle densities of each species $\rho_{1}(\mathbf{r})$ and $\rho_{2}(\mathbf{r})$, or, alternatively, by the total density $\rho(\mathbf{r}) \equiv \rho_{1}(\mathbf{r})$ $+\rho_{2}(\mathbf{r})$ and the mole fraction $x(\mathbf{r})$, which we take to be the concentration of component $2\left(x \equiv x_{2}=\rho_{2} / \rho\right)$. A fundamental result of the density-functional theory is that the Helmholtz free energy $\mathcal{F}\left[\rho_{1}, \rho_{2}\right]$ is a unique functional of the densities and is minimized (at constant average density) by the correct equilibrium densities.

The free energy can be separated into ideal and excess terms as follows:

$$
\mathcal{F}\left[\rho_{1}, \rho_{2}\right]=\mathcal{F}_{\text {id }}\left[\rho_{1}, \rho_{2}\right]+\mathcal{F}_{\text {ex }}\left[\rho_{1}, \rho_{2}\right],
$$

where the ideal part is simply the sum of the ideal free energies of the individual components

$$
\mathcal{F}_{\text {id }}\left[\rho_{1}, \rho_{2}\right]=\beta^{-1} \sum_{i=1}^{2} \int d \mathbf{r} \rho_{i}(\mathbf{r})\left[\ln \Lambda_{i}^{3} \rho_{i}(\mathbf{r})-1\right],
$$

with $\Lambda_{i}$ being the thermal wavelength of component $i$. An analytic expression for the excess free energy is not available and, in practice, this term must be approximated. In general, a density-functional theory is a procedure for the specification of this excess free-energy functional. One natural starting point for the development of such theories is the hierarchy of $n$-particle direct correlation functions $c_{i \ldots j}^{(n)}$ defined as functional derivatives of $\mathcal{F}_{\text {ex }}$ with respect to the densities

$$
\begin{array}{r}
c_{i \cdots j}^{(n)}\left(\mathbf{r}_{1}, \ldots, \mathbf{r}_{n} ; \rho_{1}, \rho_{2}\right)=-\beta \frac{\delta^{n} \mathcal{F}_{\mathrm{ex}}\left[\rho_{1}, \rho_{2}\right]}{\delta \rho_{i}\left(\mathbf{r}_{1}\right) \cdots \delta \rho_{j}\left(\mathbf{r}_{n}\right)}, \\
i, \ldots, j=1,2
\end{array}
$$

Most theories to date are constructed so that Eq. (3) is exact in the homogeneous fluid limit-at least for $n=2$. Of such theories, the WDA and MWDA have been perhaps the most successful for single-component systems. In what follows we discuss extensions of these theories to multicomponent systems - the original single component theories can be recovered in the limit that the mole fraction of one species becomes unity. 
Denton and Ashcroft generalize the WDA to binary mixtures by writing the WDA excess free energy in the form [11]

$$
\begin{aligned}
\mathcal{F}_{\text {ex }}\left[\rho_{1}, \rho_{2}\right]= & \int d \mathbf{r} \rho_{1}(\mathbf{r}) \psi\left(\bar{\rho}^{(1)}(\mathbf{r}), x\right) \\
& +\int d \mathbf{r} \rho_{2}(\mathbf{r}) \psi\left(\bar{\rho}^{(2)}(\mathbf{r}), x\right),
\end{aligned}
$$

where $\psi(\rho, x)$ is the excess free energy per particle of the corresponding uniform mixture, and $x$ represents the global average concentration of the nonuniform mixture. The two different total weighted densities $\bar{\rho}^{(1)}(\mathbf{r})$ and $\bar{\rho}^{(2)}(\mathbf{r})$ are defined as weighted averages of the physical densities with respect to weight functions $w_{i j}$ according to

$$
\bar{\rho}^{(i)}(\mathbf{r}) \equiv \sum_{j=1}^{2} \int d \mathbf{r}^{\prime} \rho_{j}\left(\mathbf{r}^{\prime}\right) w_{i j}\left(\left|\mathbf{r}-\mathbf{r}^{\prime}\right| ; \bar{\rho}^{(i)}(\mathbf{r}), x\right), \quad i=1,2 .
$$

The weight functions must be normalized

$$
\int d \mathbf{r}^{\prime} w_{i j}\left(\left|\mathbf{r}-\mathbf{r}^{\prime}\right| ; \rho\right)=1, \quad i, j=1,2
$$

and such that the approximate $\mathcal{F}_{\text {ex }}$ exactly satisfies Eq. (3) for $n=2$ in the uniform fluid limit

$$
\begin{array}{r}
c_{i j}\left(\left|\mathbf{r}_{1}-\mathbf{r}_{2}\right| ; \rho_{1}, \rho_{2}\right)=-\beta \lim _{\rho(\mathbf{r}) \rightarrow \rho}\left[\frac{\delta^{2} \mathcal{F}_{\text {ex }}\left[\rho_{1}, \rho_{2}\right]}{\delta \rho_{i}\left(\mathbf{r}_{2}\right) \delta \rho_{j}\left(\mathbf{r}_{1}\right)}\right], \\
i, j=1,2 .
\end{array}
$$

In order to compute the weight functions, one now has to solve the system of three nonlinear differential equations [11].

The MWDA is generalized by Denton and Ashcroft in the same spirit with

$$
\mathcal{F}_{\text {ex }}\left[\rho_{1}, \rho_{2}\right]=N_{1} \psi\left(\bar{\rho}^{(1)}, x\right)+N_{2} \psi\left(\bar{\rho}^{(2)}, x\right),
$$

where the weighted densities $\bar{\rho}^{(1)}$ and $\bar{\rho}^{(2)}$ are positionindependent and defined as

$$
\begin{array}{r}
\bar{\rho}^{(i)} \equiv \frac{1}{N_{i}} \sum_{j=1}^{2} \int d \mathbf{r} d \mathbf{r}^{\prime} \rho_{i}(\mathbf{r}) \rho_{j}\left(\mathbf{r}^{\prime}\right) w_{i j}\left(\left|\mathbf{r}-\mathbf{r}^{\prime}\right| ; \bar{\rho}^{(i)}, x\right), \\
i=1,2 .
\end{array}
$$

As in the single-component case, the MWDA is much less computationally demanding, so Denton and Ashcroft have used this approximation to compute freezing conditions of the binary hard-sphere mixtures with different diameter ratios. Their results for $\alpha>0.85$ closely follow those obtained in the simulations by Kranendonk and Frenkel [12].

Nevertheless, the binary mixture WDA of Denton and Ashcroft defined by Eqs. (4) and (5) cannot be used to study any binary system with extended spatial variations of average composition. The problems are best illustrated by the example of a planar crystal-fluid interface. The excess free energy $\mathcal{F}_{\text {ex }}$ in Eq. (4) and, therefore, the weight functions $w_{i j}$ in Eq. (5) are defined to depend explicitly on the global average concentration $x$. Obviously, this quantity cannot be uniquely defined for the interfacial system, where it has to be equal to the crystal coexistence values $x^{c}$ on the one side of the interface and to the fluid value $x^{f}$ on the other side. Mathematically, the WDA equations for $w_{i j}(k ; \rho, x)$ derived by Denton and Ashcroft [11] contain terms proportional to $\delta_{k, 0}$, so that the computed weight functions are discontinuous in Fourier space at $k=0$. This means that one cannot recover the bulk crystal or fluid properties in the regions remote from the interface (in the limit $z \rightarrow \pm \infty$, where $z$ is the axis perpendicular to the interfacial plane).

In order to circumvent these difficulties, we propose an extension of the WDA to binary mixtures in which weighted concentrations $\bar{x}^{(1)}(\mathbf{r})$ and $\bar{x}^{(2)}(\mathbf{r})$ are introduced in addition to the weighted densities. The weighted concentrations replace the average concentration $x$ in Eq. (4) and are defined according to

$$
\begin{array}{r}
\bar{x}^{(i)}(\mathbf{r}) \equiv \frac{1}{\bar{\rho}^{(i)}(\mathbf{r})} \int d \mathbf{r}^{\prime} \rho_{2}\left(\mathbf{r}^{\prime}\right) w_{i 2}\left(\left|\mathbf{r}-\mathbf{r}^{\prime}\right| ; \bar{\rho}^{(i)}(\mathbf{r}), \bar{x}^{(i)}(\mathbf{r})\right), \\
i=1,2 . \quad(10)
\end{array}
$$

It is more convenient to write this extension scheme in terms of the weighted densities of individual species $\bar{\rho}_{j}^{(i)}(\mathbf{r})$ defined as

$\bar{\rho}_{j}^{(i)}(\mathbf{r}) \equiv \int d \mathbf{r}^{\prime} \rho_{j}\left(\mathbf{r}^{\prime}\right) w_{i j}\left(\left|\mathbf{r}-\mathbf{r}^{\prime}\right| ; \bar{\rho}_{1}^{(i)}(\mathbf{r}), \bar{\rho}_{2}^{(i)}(\mathbf{r})\right), \quad i, j=1,2$

which are input into the excess free-energy expression,

$$
\begin{aligned}
\mathcal{F}_{\text {ex }}\left[\rho_{1}, \rho_{2}\right]= & \int d \mathbf{r} \rho_{1}(\mathbf{r}) \psi\left(\bar{\rho}_{1}^{(1)}(\mathbf{r}), \bar{\rho}_{2}^{(1)}(\mathbf{r})\right) \\
& +\int d \mathbf{r} \rho_{2}(\mathbf{r}) \psi\left(\bar{\rho}_{1}^{(2)}(\mathbf{r}), \bar{\rho}_{2}^{(2)}(\mathbf{r})\right),
\end{aligned}
$$

where $\psi\left(\rho_{1}, \rho_{2}\right)$ is the same excess free energy per particle as in Eq. (4), but expressed in variables $\rho_{1}$ and $\rho_{2}$.

Substitution of $\mathcal{F}_{\text {ex }}$ from Eq. (12) into Eq. (7) leads explicitly to the following set of equations for the three weight functions in Fourier space:

$$
\begin{aligned}
-\beta^{-1} c_{11}(k)=2 & \frac{\partial \psi}{\partial \rho_{1}} w_{11}(k)+\frac{\partial^{2} \psi}{\partial \rho_{1}^{2}}\left[\rho_{1} w_{11}^{2}(k)+\rho_{2} w_{12}^{2}(k)\right] \\
+ & 2 \frac{\partial \psi}{\partial \rho_{1}}\left[\rho_{1} w_{11}(k) \frac{\partial w_{11}(k)}{\partial \rho_{1}}\right. \\
+ & \left.\rho_{2} w_{12}(k) \frac{\partial w_{12}(k)}{\partial \rho_{1}}\right], \\
-\beta^{-1} c_{12}(k)= & \left.\frac{\partial \psi}{\partial \rho_{1}}+\frac{\partial \psi}{\partial \rho_{2}}\right) w_{12}(k)+\frac{\partial^{2} \psi}{\partial \rho_{1} \partial \rho_{2}} \\
& \times\left[\rho_{1} w_{11}(k)+\rho_{2} w_{22}(k)\right] w_{12}(k) \\
& +\frac{\partial \psi}{\partial \rho_{1}}\left[\rho_{1} w_{12}(k) \frac{\partial w_{11}(k)}{\partial \rho_{2}}\right. \\
& \left.+\rho_{2} w_{22}(k) \frac{\partial w_{12}(k)}{\partial \rho_{2}}\right]+\frac{\partial \psi}{\partial \rho_{2}}\left[\rho_{1} w_{11}(k)\right. \\
& \left.\times \frac{\partial w_{12}(k)}{\partial \rho_{1}}+\rho_{2} w_{12}(k) \frac{\partial w_{22}(k)}{\partial \rho_{1}}\right],
\end{aligned}
$$




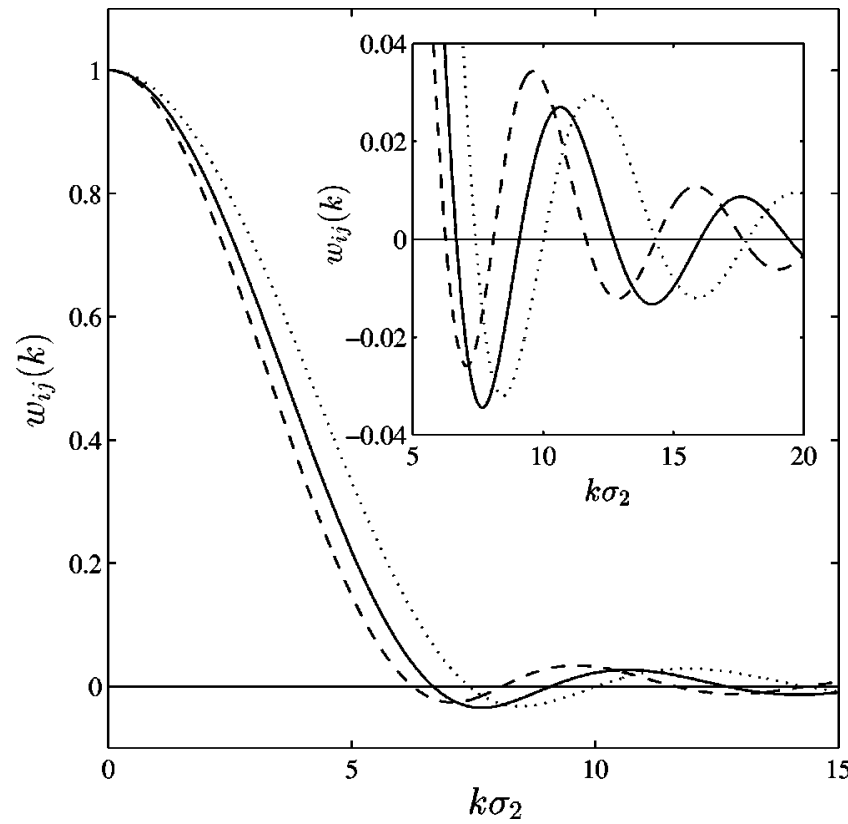

FIG. 1. WDA weight functions $w_{i j}(k)$ for a binary hard-sphere mixture. The system parameters are $\alpha=0.8, x=0.7$, and $\rho$ $=1.1 \sigma_{2}^{-3}$. The dotted, solid, and dashed curves correspond to $w_{11}$, $w_{12}$, and $w_{22}$, respectively.

$$
\begin{aligned}
-\beta^{-1} c_{22}(k)= & 2 \frac{\partial \psi}{\partial \rho_{2}} w_{22}(k)+\frac{\partial^{2} \psi}{\partial \rho_{2}^{2}}\left[\rho_{1} w_{12}^{2}(k)+\rho_{2} w_{22}^{2}(k)\right] \\
& +2 \frac{\partial \psi}{\partial \rho_{2}}\left[\rho_{1} w_{12}(k) \frac{\partial w_{12}(k)}{\partial \rho_{2}}\right. \\
& \left.+\rho_{2} w_{22}(k) \frac{\partial w_{22}(k)}{\partial \rho_{2}}\right]
\end{aligned}
$$

The normalization of the weight functions ensures that at $k=0$ Eqs. (13) $-(15)$ correctly reduce to the compressibility rules for a binary mixture [14],

$$
-\beta^{-1} c_{i j}\left(k=0 ; \rho_{1}, \rho_{2}\right)=\frac{\partial \psi}{\partial \rho_{i}}+\frac{\partial \psi}{\partial \rho_{j}}+\rho \frac{\partial^{2} \psi}{\partial \rho_{i} \partial \rho_{j}}, \quad i, j=1,2 .
$$

Unlike the original generalization of Denton and Ashcroft [11], the above equations do not contain terms proportional to $\delta_{k, 0}$, and are thus continuous functions of $k$.

In order to calculate the weight functions from Eqs. (13)(15), we need to specify $c_{i j}(k)$, the Fourier transforms of the uniform direct correlation functions, and $\psi\left(\rho_{1}, \rho_{2}\right)$, the Helmholtz free energy per particle. As an example, we have calculated the weight functions for the binary hard-sphere mixture with diameter ratio $\alpha \equiv \sigma_{1} / \sigma_{2}=0.8$ and use as input the analytical expressions for a uniform binary mixture within the Percus-Yevick approximation [13,14].

Equations (13)-(15) are a system of nonlinear differential equations. We use an iterative scheme, which has satisfactory convergence for all values of $k$ and density. We start by assuming that $w_{12}$ and all derivatives of $w_{i j}$ are zero and solve the quadratic equations for $w_{11}$ and $w_{22}$. Then we estimate the derivatives of $w_{11}$ and $w_{22}$ using finite differences and solve the equation for $w_{12}$, which is linear in $w_{12}$. Finally, we estimate the derivative of $w_{12}$. After five to ten

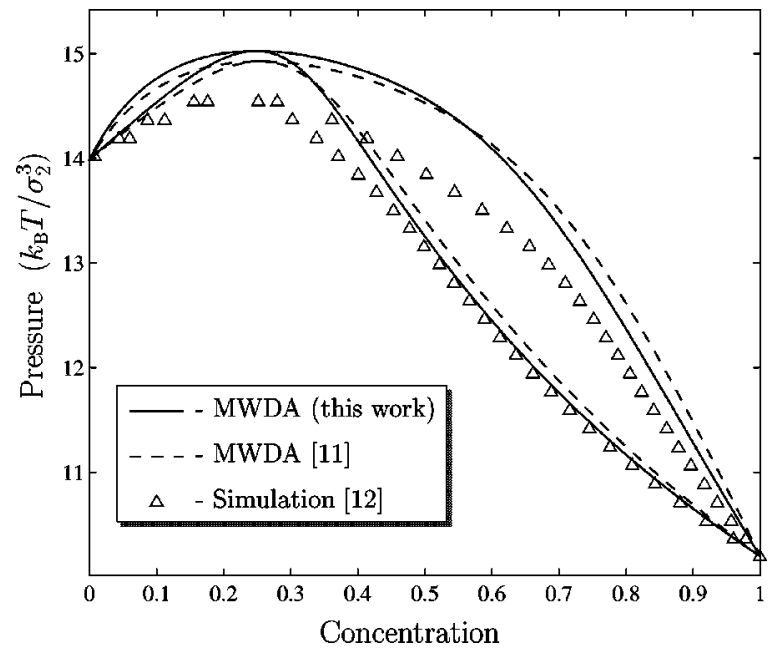

FIG. 2. Comparison of binary hard-sphere crystal-melt phase diagrams computed with the original MWDA by Denton and Ashcroft [11] (dashed lines) and our extension of the MWDA (solid lines). Also shown are the rescaled simulation data from Ref. [12] (see text for explanation).

iterations the solution converges to within five significant digits. Figure 1 shows $w_{i j}(k)$ computed for the binary hardsphere fluid with diameter ratio $\alpha=0.8$, concentration $x$ $=0.7$, and total density $\rho=1.1 \sigma_{2}^{-3}$. All three functions smoothly approach unity when $k \rightarrow 0$, thus satisfying the normalization conditions and allowing application of the WDA to binary systems with spatially varying characteristics.

In order to compare our extension of the WDA to mixtures with that of Denton and Ashcroft [11], we repeat the freezing calculations for a binary hard-sphere mixture using the MWDA consistent with the extension of the WDA presented earlier. The excess free-energy functional is written as

$$
\mathcal{F}_{\text {ex }}\left[\rho_{1}, \rho_{2}\right]=N_{1} \psi\left(\bar{\rho}_{1}^{(1)}, \bar{\rho}_{2}^{(1)}\right)+N_{2} \psi\left(\bar{\rho}_{1}^{(2)}, \bar{\rho}_{2}^{(2)}\right),
$$

where the weighted densities $\bar{\rho}_{j}^{(i)}$ are defined as

$$
\begin{array}{r}
\bar{\rho}_{j}^{(i)} \equiv \frac{1}{N_{i}} \int d \mathbf{r} d \mathbf{r}^{\prime} \rho_{i}(\mathbf{r}) \rho_{j}\left(\mathbf{r}^{\prime}\right) w_{i j}\left(\left|\mathbf{r}-\mathbf{r}^{\prime}\right| ; \bar{\rho}_{1}^{(i)}, \bar{\rho}_{2}^{(i)}\right), \\
i, j=1,2 .
\end{array}
$$

This extension of the MWDA yields the following equations for the weight function $w_{i j}(k)$ in Fourier representation:

$-\beta^{-1} c_{i j}(k)=\left(\frac{\partial \psi}{\partial \rho_{i}}+\frac{\partial \psi}{\partial \rho_{j}}\right) w_{i j}(k)+\delta_{k, 0} \rho \frac{\partial^{2} \psi}{\partial \rho_{i} \partial \rho_{j}}, \quad i, j=1,2$

where $\rho=\rho_{1}+\rho_{2}$ is the total density. Agreement with the compressibility rules given by Eq. (16) is obvious.

Comparison between the freezing parameters obtained within our extension of the MWDA and that of Denton and Ashcroft is shown in Figs. 2-4. Figure 2 presents the pressure-concentration phase diagrams together with the simulation results of Kranendonk and Frenkel [12], which are scaled down by a factor of 0.871 in order to match the transition parameters in the single-component limit $(x=0$ and 1). The scaling compensates for the discrepancies between theory and simulation in the freezing parameters of the one-component system and helps to emphasize the general 

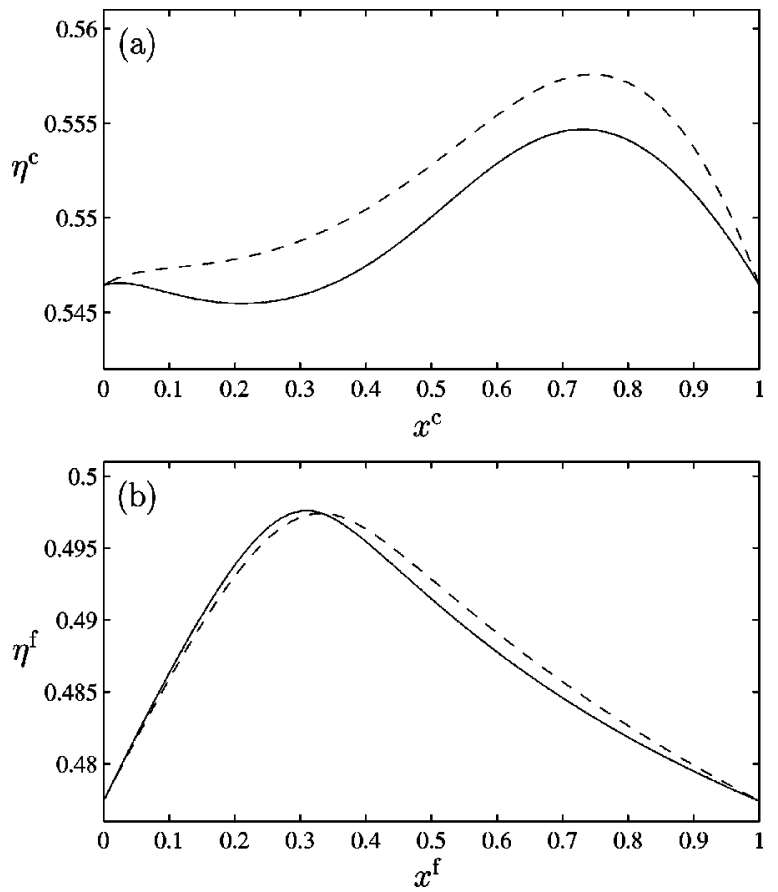

FIG. 3. (a) Packing fraction in the crystal phase at coexistence. Dashed lines represent the results of the original MWDA and solid lines represent the results from our extension of the MWDA. (b) Packing fraction in the fluid phase at coexistence.

features of the binary-system phase diagram. Overall, the two extensions of the MWDA produce very similar phase diagrams and reproduce fairly well qualitative behavior of the simulation results.

Figure 3 compares the crystal and fluid packing fractions at coexistence. The packing fractions in the crystal phase differ for the two extensions much more than the fluid packing fractions.

Figure 4 shows the Lindemann parameters for the two types of spheres. In both cases, the smaller spheres have larger Lindemann parameters for all values of concentration. The Lindemann parameters for the two extensions of the

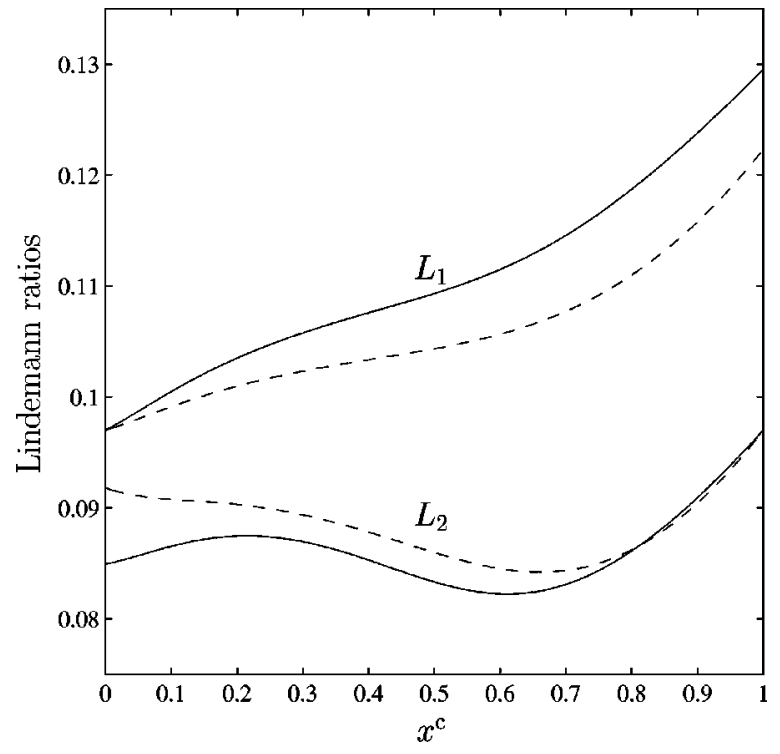

FIG. 4. Lindemann parameters for the two types of spheres calculated according to the original MWDA (dashed lines) and our extension (solid lines).

MWDA coincide for larger spheres at $x=1$, and for smaller spheres at $x=0$, where the single-component limit is recovered for the remaining type of spheres.

In conclusion, we have constructed an extension of the weighted-density approximation (WDA) of Curtin and Ashcroft [2] to multicomponent systems. This density-functional theory is more general than a previous extension by Denton and Ashcroft [11] in that it preserves the local character of the weighted densities and thus allows its application to systems with extended spatial variations in average composition, such as solid-liquid interfaces.

This research was supported by the National Science Foundation under Grant No. CHE-950281. The authors also thank the Kansas Institute for Theoretical and Computational Science (KITCS) and the Kansas Center for Advanced Scientific Computing (KCASC) for support and computing resources.
[1] R. Evans, in Fundamentals of Inhomogeneous Fluids, edited by D. Henderson (Dekker, New York, 1992), p. 85.

[2] W. A. Curtin and N. W. Ashcroft, Phys. Rev. A 32, 2909 (1985).

[3] W. A. Curtin and N. W. Ashcroft, Phys. Rev. Lett. 56, 2775 (1986).

[4] W. A. Curtin and K. Runge, Phys. Rev. A 35, 4755 (1987).

[5] R. Ohnesorge, H. Löwen, and H. Wagner, Phys. Rev. E 50, 4801 (1995).

[6] D. M. Kroll and B. B. Laird, Phys. Rev. A 42, 4806 (1990).

[7] A. R. Denton and N. W. Ashcroft, Phys. Rev. A 39, 4701
(1989).

[8] B. B. Laird and D. M. Kroll, Phys. Rev. A 42, 4810 (1990).

[9] B. B. Laird, J. Chem. Phys. 97, 2699 (1992).

[10] D. W. Marr and A. P. Gast, Phys. Rev. E 47, 1212 (1993).

[11] A. R. Denton and N. W. Ashcroft, Phys. Rev. A 42, 7312 (1990).

[12] W. G. T. Kranendonk and D. Frenkel, J. Phys.: Condens. Matter 1, 7735 (1989).

[13] J. L. Liebowitz, Phys. Rev. 133, A895 (1964).

[14] N. W. Ashcroft and D. C. Langreth, Phys. Rev. 156, 685 (1967); 166, 934(E) (1967). 